\title{
Association between fast-food consumption and lifestyle characteristics in Greek children and adolescents; results from the EYZHN (National Action for Children's Health) programme
}

\author{
Konstantinos D Tambalis ${ }^{1}$, Demosthenes B Panagiotakos ${ }^{1}$, Glyceria Psarra ${ }^{1}$ and \\ Labros S Sidossis ${ }^{1,2, *}$ \\ 'Department of Nutrition and Dietetics, School of Health Science \& Education, Harokopio University, Athens, \\ Greece: ${ }^{2}$ Department of Kinesiology and Health, Rutgers University, New Brunswick, NJ 08901, USA
}

Submitted 4 February 2018: Final revision received 24 August 2018: Accepted 13 September 2018: First published online 160 October 2018

\begin{abstract}
Objective: To examine the prevalence of fast-food consumption and the association between fast food and lifestyle factors in a representative sample of children and adolescents.

Design: Cross-sectional, observational study. Fast-food consumption and dietary habits were evaluated using questionnaires (KIDMED index). Anthropometric and physical fitness measurements were obtained by trained investigators. Physical activity (PA) status, sedentary activities and sleeping habits were assessed through self-completed questionnaires.

Setting: Greece.

Subjects: Population data derived from a school-based health survey (EYZHN programme) carried out in 2015 on 177091 ( $51 \%$ boys) children aged 8-17 years. Results: A greater proportion of boys $v$. girls $(23.3 v .15 .7 \%, P<0.001)$ and of adolescents $v$. children $(26.9 v .17 \cdot 1 \%, P<0.001)$ reported they consume fast foods $>1$ time/week. Frequent fast-food consumption was strongly correlated with unhealthy dietary habits such as skipping breakfast and consuming sweets/candy regularly. Adjusting for several covariates, insufficient dietary habits, insufficient $(<8-9 \mathrm{~h} / \mathrm{d})$ sleep, inadequate PA levels and increased screen time increased the odds $(95 \% \mathrm{CI})$ of being a frequent fast-food consumer by $77 \%(0 \cdot 218,0 \cdot 234), 30 \%$ (1.270, 1.338), 94\% (1.887, 1.995) and 32\% (1.287, 1.357), respectively. Being overweight/obese or centrally obese did not correlate with frequency of fast-food consumption.

Conclusions: Frequent fast-food consumption was associated with an unhealthy lifestyle profile among children and adolescents. The findings support the development of interventions to help children adopt healthier dietary habits.
\end{abstract}

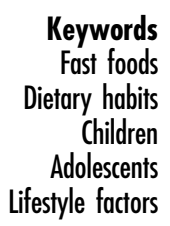

Childhood and adolescence are critical periods for the development of healthy or unhealthy dietary patterns which usually track into adulthood ${ }^{(1)}$. The traditional Mediterranean diet is characterized by increased consumption of fruits, vegetables, cereals, legumes, nuts and olive oil ${ }^{(2)}$. Numerous studies have shown that this type of diet has advantageous effects against cardiovascular, metabolic and mental diseases ${ }^{(3,4)}$. On the other hand, poor dietary habits may predispose to the development of type 2 diabetes, CVD, obesity and decreased immunity, even in schoolchildren ${ }^{(5)}$. A fast food is defined as '[an] easily prepared processed food served in snack bars and restaurants as a quick meal or to be taken away'; snacks and canned foods may also categorized as fast foods. Fast foods are often served in large portions and contain high levels of energy, fat, salt and sugar, along with low levels of fibre and micronutrients ${ }^{(6)}$. Poti et al. speculated that among US schoolchildren fast-food consumers had higher intakes of sugar-sweetened beverages and fried potatoes and lower intakes of vegetables, fruits and low-fat mixed dishes than non-consumers ${ }^{(7)}$. It is considered that frequent (more than twice weekly) fast-food consumption is strongly associated with adverse health outcomes (e.g. type 2 diabetes, obesity, dyslipidaemia) ${ }^{(8,9)}$. A review study concluded that high consumption of fast foods was associated with increased risk of development of CVD and metabolic syndrome ${ }^{(10)}$. Also, fast-food consumption has been connected to elevated intakes of unhealthy fats, 
sugar and salt, which contribute to high energy densities and glycaemic loads, and exposes children to unnecessarily large portion sizes ${ }^{(11)}$. Moreover, recent reports from fifth grade students suggested that increased fastfood consumption may correlate with lower levels of academic achievement ${ }^{(12)}$. Previous studies focusing on the association between obesity status and fast-food intake in children and adolescents reported inconsistent results $^{(7,13-15)}$. The association of sedentary lifestyle (e.g. screen time, sleeping hours $)^{(16-23)}$, physical activity $(\mathrm{PA})^{(24-27)}$ and physical fitness $(\mathrm{PF})^{(28)}$ with fast-food consumption has been investigated among schoolchildren; however, most studies have explored the association of each factor separately and in specific age groups.

To the best of our knowledge, the relationship between fast-food consumption and the lifestyle profile in Greek school-age children and adolescents is missing. Thus, we aimed to add to the literature by the examining the frequency of fast-food consumption outside the home and the association between fast-food consumption and several lifestyle characteristics using data from a large, population-representative study in Greek schoolchildren aged 8-17 years. We hypothesized that frequent fast-food consumption outside the home would be significantly associated with an unhealthy lifestyle profile as assessed through dietary habits, PA, sedentary activities and PF.

\section{Methods}

\section{Participants}

Population-based, representative data were derived from a nationwide school-based health survey (EYZHN programme) in Greece, under the auspices of the Ministry of Education. Specifically, anthropometric, nutrition, PA, sedentary habits and PF data along with information on age and gender were collected from March 2015 to May 2015. In total, 232401 ( $51 \%$ boys and 49\% girls) children and adolescents aged 8-17 years from elementary (ages 8 to 12 years) and middle (ages 13 to 17 years) public and private schools agreed to participate in the study (participation rate was almost $40 \%$ of the total population). Parents were informed in writing about the purposes of this school health survey.

\section{Assessment of demographic and antbropometric measurements}

Demographic information of students (e.g. school, class, gender and date of birth) was obtained from each school's principal. Children's height, weight and waist circumference were measured in the morning, using a standardized procedure. BMI status (normal weight, overweight, obese) was classified using the International Obesity Task Force's age- and gender-specific BMI cut-off criteria $^{(29)}$. For the purposes of the present study, we have compared normal-weight with overweight (including obese) participants. The ratio of waist circumference (in centimetres) to height (in centimetres) was calculated and central obesity was defined when this ratio was $\geq 0 \cdot 5^{(30)}$. Physical Education (PE) professionals performed all anthropometric measurements. As the measurements were included in an obligatory school curriculum, verbal informed consent by the students was considered sufficient.

\section{Assessment of physical fitness levels}

The Euro-fit PF test battery was used to evaluate children's PF levels ${ }^{(31)}$. The battery consists of five tests: (i) a multistage $20 \mathrm{~m}$ shuttle run test, to estimate aerobic performance; (ii) a maximum $10 \times 5 \mathrm{~m}$ shuttle run test, to evaluate speed and agility; (iii) sit-ups performed over $30 \mathrm{~s}$, to measure the endurance of the abdominal and hipflexor muscles; (iv) a standing long jump, to evaluate lower-body explosive power; and (v) a sit-and-reach test, to measure flexibility. All five fitness tests were administered during the PE class by PE professionals, who were instructed through a detailed manual of operations and followed a standardized procedure of measurements to minimize the inter-rater variability among schools.

\section{Assessment of dietary habits}

Participating children's dietary, PA and sedentary habits were recorded via the use of an electronic questionnaire that was completed at school with the assistance of their class and/or Information Technology teachers. Students' dietary habits were assessed using the KIDMED (Mediterranean Diet Quality Index for children and adolescents) ${ }^{(2)}$. This index contains sixteen yes or no questions, including dietary habits that are in accordance with the principles of the Mediterranean diet pattern and the general dietary guidelines for youth, and habits that undermine them. Questions denoting a negative connotation with respect to a high-quality diet are assigned a value of -1 , while those with a positive aspect are assigned a value of +1 . Thus, the total KIDMED score ranges from 0 to 12 and is classified into three levels: $\geq 8$, suggesting an optimal adherence to the Mediterranean diet (sufficient dietary habits); 4-7, suggesting an average adherence to the Mediterranean diet and an improvement needed to adjust dietary intake to guidelines (relatively sufficient dietary habits); and $\leq 3$, suggesting a low adherence to the Mediterranean diet and generally a low diet quality (insufficient dietary habits).

The main outcome measure was children's fast-food intake. To measure children's fast-food intake, we asked the question, 'Do you go $>1$ time/week to a fast-food restaurant (e.g. hamburger, pizza, etc.)?' Responses to this question were 'no' or 'yes'. The frequencies ' $\leq 1$ time/ week' and ' $>1$ time/week' were incorporated for analysis 
as a previous study suggested that the health risks connected to fast foods come about in those with frequent consumption (more than once weekly) ${ }^{(8)}$.

\section{Assessment of self-reported physical activity and sedentary time}

Patterns of PA were also self-reported. The questionnaire applied has been previously validated ${ }^{(32)}$ and included simple, closed-type questions regarding children's frequency, time and intensity of participation in: (i) schoolrelated PA (including activity during PE classes); (ii) organized sports activities; and (iii) PA during leisure time. The frequency of all reported activities was multiplied by the minutes of moderate-to-vigorous PA and then divided by 7 to obtain the mean daily time children engaged in moderate-to-vigorous PA. Children who participated in moderate-to-vigorous PA for at least for $60 \mathrm{~min} / \mathrm{d}$ were considered as meeting the recommendations for PA.

Daily time spent in sedentary activities (e.g. television viewing, use of Internet for non-study reasons, playing on computer or/and games console) was also calculated for each student (via multiplying the weekly frequency of participation with the duration per bout of participation in sedentary activities, and then dividing by 7). Using the threshold of $2 \mathrm{~h} / \mathrm{d}$ as per current guidelines ${ }^{(33)}$, students were classified as sedentary if they exceeded the recommended daily time spent in sedentary activities $(>2 \mathrm{~h} / \mathrm{d})$; otherwise, as not sedentary $(\leq 2 \mathrm{~h} / \mathrm{d})$.

Moreover, sleep time was assessed through selfreported recordings. Based on the Consensus Statement of the American Academy of Sleep Medicine, we classified as meeting the recommendations of sufficient sleep those children (aged 6-12 years) who were sleeping at least $9 \mathrm{~h} / \mathrm{d}$ and those adolescents (aged 13-17 years) who were sleeping at least $8 \mathrm{~h} / \mathrm{d}$. Children and adolescents who were sleeping less than the recommended hours were classified as having insufficient sleep ${ }^{(34)}$.

\section{Etbical approval}

Ethical approval for the health survey was granted by the Ethical Review Board of the Ministry of Education and the Ethical Review Committee of Harokopio University.

\section{Data analysis}

Descriptive statistics are expressed as mean and SD, or as frequency and percentage. The $\chi^{2}$ test was applied to evaluate associations between categorical variables and Student's $t$ test to evaluate differences in mean values of normally distributed variables. To assess the potential effect of several dietary habits on ' $\leq 1$ time/week $v$. ' $>1$ time/week' fast-food consumption, binary logistic regression analysis was implemented and OR with corresponding $95 \%$ CI were calculated, adjusted for confounders. Furthermore, aiming to assess the potential effect of several demographic and lifestyle factors on the frequency of fast-food consumption, hierarchical binary logistic regression analysis was implemented and OR with corresponding $95 \%$ CI were calculated to obtain adjusted associations of covariates while controlling for confounding. The Hosmer and Lemeshow goodness-of-fit test was calculated to evaluate the model's goodness-of-fit and residual analysis was implicated using $d \beta$, leverage and Cook's distance $D$ statistics to identify outliers and influential observations. Finally, discriminant analysis was used to explore the strength of each component in relation to the outcome. All statistical analyses were performed using the statistical software package IBM SPSS Statistics version 23.0 for Windows. Statistical significance level from twosided hypotheses was set at $P<0 \cdot 05$.

\section{Results}

Data were analysed only for those children who filled out their questionnaire (i.e. 177091 children). Basic descriptive statistics of the total sample and by gender of participants in the school-based health survey (EYZHN programme) are presented in Table 1. A greater proportion of boys than girls reported that they consume fast foods $>1$ time/week $(23.3 v .15 .7 \%, P<0.001)$. Significant differences between boys and girls were found in anthropometric variables (e.g. BMI, waist circumference), dietary habits (e.g. KIDMED index), PA, screen time and PF measurements (all $P<0 \cdot 001$ ). Among girls aged 8-12 years, $13 \cdot 6 \%$ consumed fast foods $>1$ time/week, while the corresponding proportion in adolescents aged 13-17 years was $22.1 \%(P<0.001)$. Among boys, 20.5 and $31.3 \%$ of children and adolescents, respectively, reported that they consume fast foods $>1$ time/week $(P<0.001)$.

Table 2 provides a description of the study participants according to fast-food consumption of $\leq 1$ time/week and $>1$ time/week. Participants from both genders and age ranges classified as frequent consumers of fast foods reported poorer dietary habits, increased screen time, sleeping less and having lower PF and PA levels in comparison to infrequent consumers from the same gender and age range (all $P<0 \cdot 05$ ).

In unadjusted multivariate binary logistic regression, skipping breakfast, consuming sweets and candy several times every day, eating pasta or rice almost every day and consuming nuts regularly increased the odds of being a frequent fast-food consumer in both genders, while eating a second fruit every day, eating pulses more than once weekly, using olive oil at home and eating two yoghurts and/or cheese $(40 \mathrm{~g})$ daily were associated with lower odds of being a frequent fast-food consumer (Table 3, model 1). After adjusting for several covariates (e.g. age, BMI, waist circumference, sleeping hours and PA levels), the food habits previously reported remained significantly associated with frequent consumption of fast foods in both 
Table 1 Baseline characteristics of participants: Greek children and adolescents aged 8-17 years from a school-based health survey (EYZHN programme), 2015

\begin{tabular}{|c|c|c|c|c|c|c|c|}
\hline & \multicolumn{2}{|c|}{ Total $(n 177091)$} & \multicolumn{2}{|c|}{ Boys ( $n 87803)$} & \multicolumn{2}{|c|}{ Girls ( $n$ 89288) } & \multirow[b]{2}{*}{$P^{\star}$} \\
\hline & Mean or $n$ & SD or $\%$ & Mean or $n$ & SD or $\%$ & Mean or $n$ & SD or $\%$ & \\
\hline Age (years), mean and SD & 9.88 & $2 \cdot 8$ & 9.91 & $2 \cdot 8$ & $9 \cdot 84$ & $2 \cdot 8$ & $<0.001$ \\
\hline Children, $8-12$ years, $n$ and $\%$ & 128134 & $100 \cdot 0$ & 65161 & $50 \cdot 9$ & 62973 & $49 \cdot 1$ & $<0.001$ \\
\hline Adolescents, $13-17$ years, $n$ and $\%$ & 48975 & $100 \cdot 0$ & 25660 & $52 \cdot 5$ & 23315 & $47 \cdot 5$ & $<0.001$ \\
\hline $\mathrm{BMI}\left(\mathrm{kg} / \mathrm{m}^{2}\right)$, mean and SD & $19 \cdot 7$ & 3.8 & $19 \cdot 8$ & 3.8 & 19.5 & 3.7 & $<0.001$ \\
\hline Waist circumference $(\mathrm{cm})$, mean and SD & $70 \cdot 4$ & $10 \cdot 7$ & $71 \cdot 6$ & $11 \cdot 1$ & $69 \cdot 2$ & $10 \cdot 2$ & $<0.001$ \\
\hline Fast-food intake $>1$ time/week, $n$ and $\%$ & 33520 & $19 \cdot 6$ & 20421 & $23 \cdot 3$ & 13099 & $15 \cdot 7$ & $<0.001$ \\
\hline KIDMED score (range 0 to 12 ), mean and SD & 6.7 & 2.4 & 6.7 & $2 \cdot 4$ & $6 \cdot 8$ & 2.4 & $<0.001$ \\
\hline Physical activity (h/week), mean and SD & $9 \cdot 4$ & 5.5 & $10 \cdot 4$ & $5 \cdot 9$ & 8.4 & $5 \cdot 2$ & $<0.001$ \\
\hline Screen time (h/week), mean and SD & 1.23 & 0.41 & 1.25 & 0.43 & 1.20 & 0.40 & $<0.001$ \\
\hline Sleeping time $(\mathrm{h} / \mathrm{d})$, mean and SD & 8.6 & 1.6 & 8.6 & 1.6 & 8.6 & 1.6 & 0.565 \\
\hline 20 m shuttle run (laps), mean and SD & $31 \cdot 1$ & $18 \cdot 6$ & $36 \cdot 2$ & $20 \cdot 6$ & $25 \cdot 4$ & $13 \cdot 9$ & $<0.001$ \\
\hline Standing long jump $(\mathrm{cm})$, mean and SD & 117 & $55 \cdot 7$ & 124 & $59 \cdot 3$ & 110 & 50.5 & $<0.001$ \\
\hline Sit and reach $(\mathrm{cm})$, mean and SD & $15 \cdot 4$ & $8 \cdot 3$ & $13 \cdot 2$ & $7 \cdot 6$ & $17 \cdot 7$ & $8 \cdot 3$ & $<0.001$ \\
\hline Sit-ups (no. in $30 \mathrm{~s}$ ), mean and SD & $19 \cdot 7$ & $5 \cdot 7$ & $20 \cdot 6$ & $5 \cdot 8$ & $18 \cdot 7$ & $5 \cdot 3$ & $<0.001$ \\
\hline $10 \times 5 \mathrm{~m}$ shuttle run $(\mathrm{s})$, mean and $\mathrm{SD}$ & 21.5 & 3.4 & $21 \cdot 0$ & 3.4 & $22 \cdot 1$ & $3 \cdot 3$ & $<0.001$ \\
\hline
\end{tabular}

KIDMED, Mediterranean Diet Quality Index for children and adolescents.

${ }^{*} P$ values for differences between boys and girls.

Table 2 Anthropometric and behavioural characteristics, according to weekly fast-food consumption and gender, of Greek children and adolescents aged 8-17 years from a school-based health survey (EYZHN programme), 2015

\begin{tabular}{|c|c|c|c|c|c|c|c|c|}
\hline & \multicolumn{4}{|c|}{ Fast-food consumption $\leq 1$ time/week } & \multicolumn{4}{|c|}{ Fast-food consumption $>1$ time/week } \\
\hline & \multicolumn{2}{|c|}{ Boys } & \multicolumn{2}{|c|}{ Girls } & \multicolumn{2}{|c|}{ Boys } & \multicolumn{2}{|c|}{ Girls } \\
\hline & Mean & SD & Mean & SD & Mean & SD & Mean & SD \\
\hline \multicolumn{9}{|l|}{ Children } \\
\hline Age (years) & $10 \cdot 3$ & 1.2 & $10 \cdot 2$ & 1.2 & $10 \cdot 2$ & 1.3 & $10 \cdot 1^{*}$ & 1.3 \\
\hline $\mathrm{BMI}\left(\mathrm{kg} / \mathrm{m}^{2}\right)$ & $19 \cdot 0$ & 3.6 & $18 \cdot 6$ & 3.5 & 18.9 & 3.6 & 18.6 & 3.5 \\
\hline Waist circumference $(\mathrm{cm})$ & $69 \cdot 1$ & $10 \cdot 2$ & $67 \cdot 7$ & $10 \cdot 2$ & 68.9 & $10 \cdot 3$ & 67.6 & $10 \cdot 3$ \\
\hline WHtR & 0.48 & 0.6 & 0.47 & 0.6 & 0.48 & 0.6 & 0.47 & 0.6 \\
\hline KIDMED score & $7 \cdot 2$ & $2 \cdot 2$ & $7 \cdot 3$ & $2 \cdot 2$ & $5 \cdot 4^{\star}$ & $2 \cdot 4$ & $5 \cdot 5^{\star}$ & $2 \cdot 4$ \\
\hline Physical activity (h/week) & $10 \cdot 7$ & $5 \cdot 8$ & $9 \cdot 8$ & $5 \cdot 1$ & 10.4 & $6 \cdot 1$ & $8.9^{*}$ & 5.6 \\
\hline Screen time (h/week) & $10 \cdot 4$ & $7 \cdot 7$ & $6 \cdot 4$ & $6 \cdot 7$ & $11.5^{\star}$ & 9.8 & $9 \cdot 7^{\star}$ & $9 \cdot 0$ \\
\hline Sleeping time $(\mathrm{h} / \mathrm{d})$ & 8.8 & 1.6 & 8.9 & 1.5 & $8 \cdot 5^{\star}$ & 1.8 & $8 \cdot 5^{\star}$ & $1 \cdot 7$ \\
\hline 20 m shuttle run (laps) & $33 \cdot 1$ & $18 \cdot 1$ & 24.9 & 13.5 & $31 \cdot 2^{*}$ & $18 \cdot 1$ & $23 \cdot 0^{*}$ & 13.0 \\
\hline Standing long jump (cm) & 119 & $52 \cdot 0$ & 110 & $47 \cdot 0$ & $115^{\star}$ & 51.9 & $105^{\star}$ & $45 \cdot 8$ \\
\hline Sit and reach $(\mathrm{cm})$ & 12.9 & $7 \cdot 2$ & $17 \cdot 1$ & $7 \cdot 8$ & $12 \cdot 5^{\star}$ & $7 \cdot 2$ & $16 \cdot 0^{\star}$ & 7.9 \\
\hline Sit-ups (no. in $30 \mathrm{~s}$ ) & $19 \cdot 8$ & 5.5 & 18.5 & $5 \cdot 3$ & $19 \cdot 0^{*}$ & 5.7 & $17 \cdot 6^{\star}$ & $5 \cdot 3$ \\
\hline $10 \times 5 \mathrm{~m}$ shuttle run $(\mathrm{s})$ & $21 \cdot 3$ & $3 \cdot 2$ & $22 \cdot 1$ & 3.2 & $21 \cdot 6^{\star}$ & 3.6 & $22 \cdot 5^{\star}$ & 3.5 \\
\hline \multicolumn{9}{|l|}{ Adolescents } \\
\hline Age (years) & 14.4 & 1.3 & 14.4 & 1.3 & $14 \cdot 7^{*}$ & $1 \cdot 3$ & $14.7^{\star}$ & 1.3 \\
\hline $\mathrm{BMl}\left(\mathrm{kg} / \mathrm{m}^{2}\right)$ & 21.6 & 3.9 & 21.1 & 3.7 & 21.6 & 3.9 & 21.2 & 3.7 \\
\hline Waist circumference $(\mathrm{cm})$ & $78 \cdot 1$ & $10 \cdot 9$ & 73.6 & 9.9 & $78 \cdot 1$ & $11 \cdot 0$ & 73.5 & 9.9 \\
\hline WHtR & 0.46 & 0.6 & 0.45 & 0.6 & 0.46 & 0.6 & 0.45 & 0.6 \\
\hline KIDMED score & 6.9 & 4.7 & 6.5 & $2 \cdot 2$ & $4.7^{*}$ & $2 \cdot 3$ & $4 \cdot 3^{*}$ & $2 \cdot 2$ \\
\hline Physical activity (h/week) & $10 \cdot 7$ & 5.7 & 7.6 & 4.7 & $10 \cdot 2^{*}$ & $6 \cdot 3$ & $6 \cdot 9^{\star}$ & $5 \cdot 1$ \\
\hline Screen time (h/week) & $10 \cdot 4$ & $9 \cdot 1$ & 9.9 & 8.8 & $14 \cdot 3^{*}$ & $10 \cdot 8$ & $14 \cdot 1^{*}$ & $10 \cdot 8$ \\
\hline Sleeping time $(\mathrm{h} / \mathrm{d})$ & $8 \cdot 2$ & 1.5 & 7.9 & 1.4 & $7 \cdot 8^{\star}$ & 1.7 & $7 \cdot 6^{\star}$ & 1.6 \\
\hline 20 m shuttle run (laps) & $49 \cdot 7$ & 23.8 & 29.5 & $15 \cdot 2$ & $49 \cdot 4$ & $24 \cdot 4$ & $28 \cdot 1^{*}$ & $15 \cdot 1$ \\
\hline Standing long jump (cm) & 140 & 73.7 & 112 & $61 \cdot 1$ & 140 & $76 \cdot 4$ & $111^{*}$ & 58.5 \\
\hline Sit and reach $(\mathrm{cm})$ & $14 \cdot 3$ & 8.6 & $20 \cdot 5$ & 9.0 & 14.0 & 8.5 & $19 \cdot 1^{*}$ & 8.9 \\
\hline Sit-ups (no. in $30 \mathrm{~s}$ ) & 23.3 & $5 \cdot 6$ & $20 \cdot 0$ & $5 \cdot 0$ & 23.2 & $5 \cdot 7$ & $19 \cdot 1^{*}$ & 4.9 \\
\hline $10 \times 5 \mathrm{~m}$ shuttle run $(\mathrm{s})$ & $19 \cdot 6$ & 3.2 & $21 \cdot 3$ & 3.0 & $19 \cdot 6$ & 3.5 & $21 \cdot 6^{*}$ & 3.5 \\
\hline
\end{tabular}

KIDMED, Mediterranean Diet Quality Index for children and adolescents; WHtR, waist-to-height ratio.

${ }^{*}$ Mean values were significant different between occasional ( $\leq 1$ time/week) and frequent $(>1$ time/week) consumers of fast foods of the same gender: $P<0.01$.

genders (Table 3, model 2). Adjustment for screen time did not change the results (Table 3, model 3).

Taking into account that frequent fast-food consumers have a worse lifestyle profile compared with infrequent consumers, stepwise logistic regression analyses (four models) in both genders were applied to investigate the possible associations of several related factors with fastfood consumption ( $\leq 1$ time/week $v .>1$ time/week). The 
Table 3 Results from logistic regression models evaluating the association of dietary habits with fast-food consumption ( $\leq 1$ time/week $v$. $>1$ time/week) among Greek children and adolescents aged 8-17 years from a school-based health survey (EYZHN programme), 2015

\begin{tabular}{|c|c|c|c|c|c|c|}
\hline \multirow[b]{2}{*}{ Predictor } & \multicolumn{2}{|c|}{ Model 1} & \multicolumn{2}{|c|}{ Model 2} & \multicolumn{2}{|c|}{ Model 3} \\
\hline & OR & $95 \% \mathrm{Cl}$ & OR & $95 \% \mathrm{Cl}$ & OR & $95 \% \mathrm{Cl}$ \\
\hline \multicolumn{7}{|l|}{ Boys } \\
\hline Skips breakfast (no $v$. yes) & 1.517 & $1.462,1.575$ & 1.532 & $1.475,1.592$ & 1.495 & $1.439,1.554$ \\
\hline Has a second fruit every day (no $v$. yes) & 0.826 & $0.798,0.855$ & 0.843 & $0.814,0.872$ & 0.861 & $0.831,0.892$ \\
\hline Has fresh or cooked vegetables $>1 / d$ (no $v$. yes) & 1.015 & $0.976,1.056$ & 1.006 & $0.975,1.042$ & 1.016 & $0.972,1.060$ \\
\hline Consumes fish at least 2-3/week (no $v$. yes) & 1.017 & $0.983,1.051$ & 1.029 & $0.995,1.065$ & 1.027 & $0.991,1.063$ \\
\hline Eats pulses $>1 /$ week (no $v$. yes) & 0.796 & $0.768,0.824$ & 0.777 & $0.749,0.806$ & 0.792 & $0.764,0.822$ \\
\hline Eats pasta/rice almost every day (no $v$. yes) & 1.315 & $1.271,1.359$ & 1.303 & $1.260,1.348$ & 1.298 & $1.254,1.343$ \\
\hline Consumes nuts at least $2-3 /$ week (no $v$. yes) & 1.177 & $1 \cdot 138,1 \cdot 217$ & $1 \cdot 188$ & $1 \cdot 148,1 \cdot 230$ & 1.193 & $1.152,1.235$ \\
\hline Uses olive oil at home (no $v$. yes) & 0.746 & $0.688,0.709$ & 0.727 & $0.669,0.789$ & 0.715 & $0.658,0.778$ \\
\hline Takes two yoghurts and/or some cheese ( $40 \mathrm{~g}$ ) daily (no $v$. yes) & 0.871 & $0.806,0.938$ & 0.862 & $0.826,0.899$ & 0.855 & $0.820,0.893$ \\
\hline Takes sweets/candy several times every day (no $v$. yes) & 3.823 & $3.686,3.924$ & 3.638 & $3.505,3.776$ & 3.358 & $3.233,3.488$ \\
\hline \multicolumn{7}{|l|}{ Girls } \\
\hline Skips breakfast (no $v$. yes) & 1.483 & $1.421,1.549$ & 1.468 & $1.405,1.534$ & 1.427 & $1.365,1.492$ \\
\hline Has a second fruit every day (no $v$. yes) & 0.803 & $0.771,0.836$ & 0.818 & $0.785,0.852$ & 0.839 & $0.804,0.874$ \\
\hline Has fresh or cooked vegetables $>1 / d$ (no $v$. yes) & 0.985 & $0.941,1.032$ & 1.001 & $0.955,1.050$ & 1.000 & $0.953,1.048$ \\
\hline Consumes fish at least $2-3 /$ week (no $v$. yes) & 1.004 & $0.965,1.045$ & 1.022 & $0.981,1.064$ & 1.025 & $0.980,1.070$ \\
\hline Eats pulses $>1 /$ week (no $v$. yes) & 0.804 & $0.772,0.839$ & 0.808 & $0.774,0.842$ & 0.823 & $0.789,0.859$ \\
\hline Eats pasta/rice almost every day (no $v$. yes) & 1.459 & $1.403,1.518$ & 1.452 & $1.394,1.511$ & 1.445 & $1.387,1.504$ \\
\hline Consumes nuts at least $2-3 /$ week (no $v$. yes) & 1.231 & $1.182,1.282$ & 1.247 & $1.197,1.300$ & 1.263 & $1.212,1.317$ \\
\hline Uses olive oil at home (no $v$. yes) & 0.717 & $0.643,0.800$ & 0.704 & $0.631,0.787$ & 0.685 & $0.613,0.767$ \\
\hline Takes two yoghurts and/or some cheese ( $40 \mathrm{~g} /$ daily) (no $v$. yes) & 0.864 & $0.822,0.907$ & 0.861 & $0.819,0.905$ & 0.863 & $0.821,0.907$ \\
\hline Takes sweets/candy several times every day (no $v$. yes) & $4 \cdot 170$ & $3.998,4.350$ & 3.904 & $3.738,4.076$ & 3.606 & $3.451,3.769$ \\
\hline
\end{tabular}

Model 1: unadjusted. Model 2: adjusted for age, BMI, waist circumference, sleeping hours and physical activity levels. Model 3: model 2 plus screen time.

initial analysis revealed that increase in age (per 1 year) increased the odds of being a frequent fast-food consumer by almost $10 \%$ in both genders, while being overweight/ obese or centrally obese did not correlate with frequency of fast-food consumption (Table 4, model 1). When the KIDMED index, sleeping status and PA levels were added in the analysis (Table 4, model 2), results related to the effect of age and obesity status did not change, while insufficient dietary habits, insufficient $(<8-9 \mathrm{~h} / \mathrm{d})$ sleeping status and inadequate (moderate-to-vigorous $\mathrm{PA}<60 \mathrm{~min} /$ d) PA levels burdened children's odds of being frequent fast-food consumers. After additional adjustment for screen time (model 3), the results revealed an unfavourable influence of increased sedentary activities on frequency of fast-food consumption. Ultimately, when PF measurements were included in the analysis (model 4), the influence of previous factors did not change significantly. In the whole population, insufficient dietary habits, insufficient $(<8-9 \mathrm{~h} / \mathrm{d})$ sleeping, inadequate PA levels and increased screen time increased the odds of being a frequent fast-food consumer by $77 \%(95 \% \mathrm{CI}$ $0.218,0.234), 30 \%$ (95\% CI $1.270,1.338), 94 \%$ (95\% CI $1 \cdot 887,1.995)$ and $32 \%(95 \%$ CI 1.287, 1.357), respectively, after adjusting for several covariates. In addition, better results in PF measurements were related to lower probabilities of being a frequent fast-food consumer, in both genders. OR of all PF indicators (except for sit and reach in boys) were small (ranged from 0.982 to 0.999$)$ but statistically significant (all $P<0 \cdot 01$ ). For example, an improvement of $10 \mathrm{~s}$ in the $10 \times 5 \mathrm{~m}$ shuttle run corresponded to a
$15 \%$ decrease in the odds of being a frequent fast-food consumer.

Discriminant analysis by gender was applied to assess whether the predictors could better distinguish those with infrequent from those who had frequent consumption of fast foods. Standardized function coefficients suggested that dietary habits $(0.85)$, screen time $(0.34)$ and PA (0.29) contributed more to distinguishing those who consume fast foods frequently from those with infrequent consumption, in both genders. The classification results showed that the model correctly predicts $77 \%$ of frequent fast-food consumers and $69 \%$ of infrequent ones.

\section{Discussion}

To the best of our knowledge, the current study is the first to report anthropometric, PF and lifestyle correlates of fastfood consumption in a Greek population-representative cohort. We used data from 177091 schoolchildren (aged 8-17 years) to obtain current, reliable, standardized and comparable findings. The main findings of our study are: (i) almost $20 \%$ of schoolchildren consumed fast foods more than once weekly; (ii) participants from both genders who were frequent consumers of fast foods presented a worse lifestyle profile; and (iii) frequent fast-food consumption was strongly associated with poor dietary habits, in both genders.

Almost $20 \%$ of the surveyed population consumed fast foods more than once weekly. These findings are in 
Table 4 Results from logistic regression models evaluating the association of anthropometric and behavioural characteristics with fast-food consumption ( $\leq 1$ time/week $v .>1$ time/week) among Greek children and adolescents aged 8-17 years from a school-based health survey (EYZHN programme), 2015

\begin{tabular}{|c|c|c|c|c|c|c|c|c|}
\hline \multirow[b]{2}{*}{ Predictor } & \multicolumn{2}{|r|}{ Model 1} & \multicolumn{2}{|r|}{ Model 2} & \multicolumn{2}{|r|}{ Model 3} & \multicolumn{2}{|r|}{ Model 4} \\
\hline & OR & $95 \% \mathrm{Cl}$ & OR & $95 \% \mathrm{Cl}$ & OR & $95 \% \mathrm{Cl}$ & OR & $95 \% \mathrm{Cl}$ \\
\hline \multicolumn{9}{|l|}{ Boys } \\
\hline Age (per 1 year) & $1 \cdot 104$ & $1.097,1.112$ & 1.092 & $1.085,1.100$ & 1.076 & $1.068,1.084$ & 1.087 & $1.075,1.099$ \\
\hline BMI group (normal weight $v$. overweight/obese) & 0.980 & $0.940,1.021$ & 0.976 & $0.926,1.029$ & 0.972 & $0.931,1.015$ & 0.982 & $0.950,1.013$ \\
\hline Central obesity (no $v$. yes) & 0.984 & $0.949,1.012$ & 0.982 & $0.957,1.009$ & 0.982 & $0.957,1.009$ & 0.992 & $0.958,1.019$ \\
\hline $\begin{array}{l}\text { KIDMED index (insufficient } v \text {. sufficient dietary } \\
\text { habits) }\end{array}$ & & & 0.210 & $0.201,0.220$ & 0.229 & $0.219,0.240$ & 0.233 & $0.220,0.247$ \\
\hline Sleeping hours (sufficient $v$. insufficient) & & & 1.325 & $1 \cdot 281,1.371$ & $1 \cdot 281$ & $1.238,1.326$ & 1.279 & $1.227,1.333$ \\
\hline Physical activity (adequate $v$. inadequate) & & & 1.283 & $1.239,1.329$ & 1.245 & $1.201,1.290$ & 1.261 & $1.207,1.318$ \\
\hline Screen time (acceptable $v$. increased) & & & & & 1.891 & $1.824,1.961$ & 1.866 & $1.786,1.950$ \\
\hline $20 \mathrm{~m}$ shuttle run (per 1 lap) & & & & & & & 0.998 & $0.997,0.999$ \\
\hline Standing long jump (per $1 \mathrm{~cm}$ ) & & & & & & & 0.999 & $0.999,0.999$ \\
\hline Sit and reach $($ per $1 \mathrm{~cm})$ & & & & & & & 1.001 & $0.998,1.003$ \\
\hline Sit-ups in $30 \mathrm{~s}$ (per 1 repetition) & & & & & & & 0.988 & $0.984,0.994$ \\
\hline $10 \times 5 \mathrm{~m}$ shuttle run (per $1 \mathrm{~s})$ & & & & & & & 1.015 & $1.008,1.021$ \\
\hline \multicolumn{9}{|l|}{ Girls } \\
\hline Age (per 1 year) & 1.094 & $1.088,1.103$ & 1.059 & $1.050,1.069$ & 1.038 & $1.028,1.047$ & 1.041 & $1.028,1.051$ \\
\hline BMI group (normal weight $v$. overweight/obese) & 0.986 & $0.950,1.021$ & 0.986 & $0.946,1.029$ & 0.982 & $0.941,1.025$ & 0.982 & $0.960,1.003$ \\
\hline Central obesity (no $v$. yes) & 1.026 & $0.976,1.072$ & 1.038 & $0.981,1.097$ & 1.019 & $0.963,1.078$ & 0.963 & $0.896,1.034$ \\
\hline $\begin{array}{l}\text { KIDMED index (insufficient } v \text {. sufficient dietary } \\
\text { habits }\end{array}$ & & & $0 \cdot 198$ & $0.188,0.208$ & 0.218 & $0.206,0.229$ & 0.229 & $0.214,0.245$ \\
\hline Sleeping hours (sufficient $v$. insufficient) & & & 1.376 & $1.322,1.433$ & 1.342 & $1.287,1.397$ & 1.402 & $1.331,1.476$ \\
\hline Physical activity (adequate $v$. inadequate) & & & 1.257 & $1.209,1.308$ & 1.219 & $1.171,1.269$ & $1 \cdot 218$ & $1.158,1.281$ \\
\hline Screen time (acceptable $v$. increased) & & & & & 1.930 & $1 \cdot 846,2 \cdot 018$ & 1.913 & $1.808,2.046$ \\
\hline $20 \mathrm{~m}$ shuttle run (per 1 lap) & & & & & & & 0.995 & $0.993,0.997$ \\
\hline Standing long jump (per $1 \mathrm{~cm}$ ) & & & & & & & 0.999 & $0.999,0.999$ \\
\hline Sit and reach $($ per $1 \mathrm{~cm})$ & & & & & & & 0.993 & $0.990,0.996$ \\
\hline Sit-ups in $30 \mathrm{~s}$ (per 1 repetition) & & & & & & & 0.982 & $0.976,0.987$ \\
\hline $10 \times 5 \mathrm{~m}$ shuttle run (per $1 \mathrm{~s})$ & & & & & & & 1.012 & $1.004,1.020$ \\
\hline
\end{tabular}

KIDMED, Mediterranean Diet Quality Index for children and adolescents.

Model 1: adjusted for age, BMI group and central obesity. Model 2: model 1 plus KIDMED index, sleeping hours and physical activity levels. Model 3: model 2 plus screen time. Model 4: model 3 plus physical fitness measurements.

accordance with a study from Adams et al. in 2015, who found that $21 \%$ of UK children ( 1.5 to 18 years old) consume take-away meals once per week or more ${ }^{(35)}$; and Cutumisu et al. in 2017, who reported that $22 \%$ of secondary Canadian students consume junk foods more than twice weekly ${ }^{(36)}$. Similarly, a review study that included data from seventeen countries concluded that $23 \%$ of children and $39 \%$ of adolescents reported frequent fast-food consumption $^{(13)}$. Among our Greek population, more boys compared with girls $(23.3 v .15 .7 \%, P<0.001)$ and more adolescents compared with children (26.9 v. $17 \cdot 1 \%$, $P<0 \cdot 001)$ reported frequent consumption of fast foods. A review of almost 273000 participants highlighted a significant increased prevalence of frequent consumption in adolescents as compared with children (39 v. 23\%, $P<0.001)^{(13)}$. Regarding gender differences, our results $(\mathrm{OR}=1.59 ; 95 \% \mathrm{CI} 1.53,1.65)$ are in agreement with those from Canadian adolescents that reported higher odds of frequent consumption associated with being a male $(\mathrm{OR}=$ 1.56 ; $95 \%$ CI $1.39,1.74)^{(36)}$. These findings can potentially be attributed to the fact that adolescents (especially males) in Greece have more autonomy and opportunities to consume fast foods than children. Also, adolescents are more likely to be under peer influence than children ${ }^{(37)}$.
Although obesity in Greek youth is of great concern, fast-food consumption did not seem to be a significant contributor of total or central obesity in our study. A review study of 6- to 7-year-old children from thirty-two countries revealed that those who consumed fast foods frequently and very frequently had higher BMI by 0.15 and $0.28 \mathrm{~kg} / \mathrm{m}^{2}(P<0.001)$, respectively, than infrequent consumers ${ }^{(13)}$. Moreover, the same review in 13- to 14 -yearold adolescents revealed that those who frequently and very frequently consumed fast foods had BMI that was lower by 0.14 and $0.22 \mathrm{~kg} / \mathrm{m}^{2}(P<0.001)$, respectively, than infrequent consumers, with the exception of males from low-income countries ${ }^{(13)}$. Our data are not comparable with the studies mentioned above as we have included children aged 8-12 years and adolescents aged 13-17 years. In accordance with our results, studies in students aged 5-11 years from New Zealand, 7-16 years from China and 2-18 years from the USA found that fast-food consumption was not associated with obesity ${ }^{(7,14,15)}$. Among several potential explanations for the conflicting results on the association between fast-food consumption and obesity may include that most studies examined only the frequency and not the quantity of fast foods or the total dietary intake and habits. 
Our results suggest that dietary habits, screen time, PA levels, sleeping time and PF are significantly associated with fast-food consumption. Specifically, participants (aged 8-17 years) who were classified as frequent fastfood consumers had $80 \%$ decreased odds of having sufficient dietary habits. Moreover, in the same population, unhealthy dietary habits such as skipping breakfast and taking sweets frequently increased the odds of being a frequent fast-food consumer. A review study in 7199 children aged 9-11 years from twelve countries has shown that unhealthy dietary patterns, including fast foods, ice cream, fried foods, potato chips, cakes, etc., are strongly related to each other ${ }^{(38)}$. In line with our findings, a study among 4466 US children suggested that fast-food consumers presented higher intakes of sugar-sweetened beverages and fried potatoes and lower intakes of vegetables, fruits and low-fat mixed dishes (OR $=1 \cdot 51 ; 95 \% \mathrm{CI}$ $1.24,1.85)$ than non-consumers ${ }^{(7)}$. Furthermore, another review concluded that fast-food consumption is a major risk factor for poorer diet quality and fat intake ${ }^{(10)}$. Surprisingly, our results revealed that pasta or rice consumption and regularly consuming nuts augmented the probabilities of frequent fast-food consumption. A study in Spanish children reported that boys preferred to consume fast foods and pasta or rice more frequently ${ }^{(39)}$. In addition, a study among 6212 US schoolchildren (4-19 years) concluded that those who ate fast foods, compared with non-consumers, tended to consume more carbohydrates, total fat and sugar-sweetened beverages and less fruits, vegetables, fibre and milk $^{(40)}$. We hypothesized that the progressive increase in unhealthy dietary habits in Greece potentially leads to consumption of meals that are easier to prepare (e.g. pasta, rice). On the other hand, healthy dietary habits such as eating a second fruit every day, eating pulses more than once weekly, using olive oil at home and taking two yoghurts and/or some cheese ( $40 \mathrm{~g}$ ) daily were associated with lower odds of being a frequent fast-food consumer. Our findings agree with reports that frequency of fast-food intake is associated with the dietary intake profile ${ }^{(7,41,42)}$.

Participants of the current study with increased screen time ( $>2 \mathrm{~h} / \mathrm{d}$ ) had higher odds of being frequent fast-food consumers by almost $90 \%$, in both genders. A study among Danish children and adolescents revealed that increased television viewing was related to unhealthy food preferences and food habits ${ }^{(16)}$. Similarly, increased time of television viewing $(>2 \mathrm{~h} / \mathrm{d})$ in Canadian children was positively associated with frequency of consumption of fast foods, independently of several covariates ${ }^{(17)}$. Also, study in about 11000 US children and adolescents proposed that each one hour increase in total screen time significantly increased intakes of low nutritional quality foods (i.e. fast foods, sugar-sweetened beverages, sweets, etc. $^{(18)}$. Children who experienced increased television viewing and food advertising were more prone to unhealthy food requests ${ }^{(19)}$.
Our findings suggest that insufficient sleep duration $(<8-9 \mathrm{~h} / \mathrm{d})$ is associated with a higher probability (OR $=$ $1 \cdot 28-1.40, P<0 \cdot 001)$ of being a frequent fast-food consumer, in both genders. In a cross-sectional study of 65212 Korean students, the authors concluded that fast-food consumption was negatively associated with sleep satisfaction $^{(20)}$. Data from the National Longitudinal Study of Adolescent Health ( $n$ 13284) revealed that short sleep duration $(<7 \mathrm{~h} /$ night $)$ was associated with increased odds of fast-food consumption (OR $=1.40, P<0 \cdot 001)^{(21)}$. Also, our results are in line with those of previous studies reporting that insufficient sleep among school students is likely to increase unhealthy eating behaviours ${ }^{(22,23)}$.

In our study, a sufficient PA level was inversely related to the odds of frequent fast-food consumption, even after adjustment for several covariates. This is in agreement with previous findings, where it has been proposed that healthy dietary patterns among children and adolescents were favourably related to PA, in both genders ${ }^{(18,24,25)}$. Moreover, physically active girls reported healthier dietary habits ${ }^{(26,27)}$. Although the OR of PF variables to predict fast-food consumers were small (ranging from 0.982 to 0.999 , all $P<0 \cdot 01$ ), the current findings proposed a trend that as performance in $\mathrm{PF}$ indicators improved, the odds of frequent fast-food consumption decreased. These results are in line with those of a study among French students which proposed a positive relationship between PF and eating habits ${ }^{(28)}$. Also, studies among adults have shown that participants with higher PF levels were more likely to adhere to dietary recommendations than their less-fit peers ${ }^{(43,44)}$. Further research is needed to confirm this relationship. Regular PA and enhanced PF constitute a healthy lifestyle and potentially these children were more likely to avoid unhealthy dietary habits (e.g. fast foods).

\section{Strengths and limitations}

The present study was performed in both children and adolescents and investigated several covariates. In Greece, secondary and primary education is required and, consequently, we had the opportunity to study a very large part of the population aged 8-17 years. The methodology used allows the direct comparison of our results with those from other similarly large and representative studies.

Limitations include methodological issues and the fact that prospective confounding factors, such as socioeconomic status and availability of fast-food restaurants, which are likely connected to fast-food consumption, have not been evaluated. In addition, the study is crosssectional so causality cannot be assigned. Fast-food consumption was defined as visiting a restaurant; fast-food intakes from other sources such as takeaway/fast foods consumed at home, fast foods cooked at home and sitdown/full-service restaurants were not considered. The children's weight status was measured using BMI age- and gender-specific cut-off points. Moreover, the records of 
PA, dietary habits, sleeping time and sedentary time were self-reported, therefore subject to desirable reporting bias. Nevertheless, participants' responses were anonymous; as a result, they had no reason to misreport. Finally, because of the large sample size, statistical significance can easily be achieved.

\section{Conclusions}

The current study found that a significant proportion of Greek schoolchildren are frequent fast-food consumers. Fast-food consumption is strongly correlated with unhealthy dietary habits, such as skipping breakfast and consuming sweets regularly, and with a worse lifestyle profile in general. Urgent actions are required to help children adopt healthier dietary habits.

\section{Acknowledgements}

Financial support: This study was supported by the Hellenic Ministry of Education and Religious Affairs, Secretariat General of Sports, OPAP S.A., Nestlé Hellas S.A. and the Department of Nutrition and Dietetics Graduate Program, Harokopio University of Athens. The funding agencies had no role in the design, analysis or writing of this article. Conflict of interest: The authors declared no conflict of interest. Authorship: All authors contributed equally to the study design and analysis. K.D.T. wrote the paper. D.B.P. and L.S.S. drafted the initial form of the manuscript. All authors commented on the manuscript draft. Etbics of buman subject participation: This study was conducted according to the guidelines laid down in the Declaration of Helsinki and all procedures involving human subjects were approved by the Ethical Review Board of the Ministry of Education and the Ethical Review Committee of Harokopio University. Parents were informed in writing about the purposes of the school health survey. As the measurements were included in an obligatory school curriculum, verbal informed consent by the students was considered sufficient.

\section{References}

1. Craigie AM, Lake AA, Kelly SA et al. (2011) Tracking of obesity-related behaviours from childhood to adulthood: a systematic review. Maturitas 70, 266-284.

2. Serra-Majem L, Ribas L, Ngo J et al. (2004) Food, youth and the Mediterranean diet in Spain. Development of KIDMED, Mediterranean Diet Quality Index in children and adolescents. Public Health Nutr 7, 931-935.

3. Martinez-Gonzalez MA\& Bes-Rastrollo M (2014) Dietary patterns, Mediterranean diet, and cardiovascular disease. Curr Opin Lipidol 25, 20-26.

4. Psaltopoulou T, Sergentanis TN, Panagiotakos DB et al. (2013) Mediterranean diet, stroke, cognitive impairment, and depression: a meta-analysis. Ann Neurol 74, 580-591.
5. Schroder H (2007) Protective mechanisms of the Mediterranean diet in obesity and type 2 diabetes. $J$ Nutr Biochem 18, 149-160.

6. Feeley A, Pettifor JM \& Norris SA (2009) Fast-food consumption among 17-year-olds in the Birth to Twenty cohort. S Afr J Clin Nutr 22, 118-123.

7. Poti JM, Duffey KJ \& Popkin BM (2014) The association of fast food consumption with poor dietary outcomes and obesity among children: is it the fast food or the remainder of the diet? Am J Clin Nutr 99, 162-171.

8. Pereira MA, Kartashov AI, Ebbeling CB et al. (2005) Fast-food habits, weight gain, and insulin resistance (the CARDIA study): 15-year prospective analysis. Lancet $\mathbf{3 6 5}$, 36-42.

9. Marlatt KL, Farbakhsh K, Dengel DR et al. (2015) Breakfast and fast food consumption are associated with selected biomarkers in adolescents. Prev Med Rep 3, 49-52.

10. Bahadoran Z, Mirmiran P \& Azizi F (2016) Fast food pattern and cardiometabolic disorders: a review of current studies. Health Promot Perspect 5, 231-240.

11. Rosenheck R (2008) Fast food consumption and increased caloric intake: a systematic review of a trajectory towards weight gain and obesity risk. Obes Rev 9, 535-547.

12. Purtell KM \& Gershoff ET (2015) Fast food consumption and academic growth in late childhood. Clin Pediatr (Phila) $\mathbf{5 4}$ 871-877.

13. Braithwaite I, Stewart AW, Hancox RJ et al. (2014) Fast-food consumption and body mass index in children and adolescents: an international cross-sectional study. BMJ Open $\mathbf{4}$, e005813.

14. Duncan JS, Schofield G, Duncan EK et al. (2008) Risk factors for excess body fatness in New Zealand children. Asia Pac J Clin Nutr 17, 138-147.

15. Zhao Y, Wang L, Xue H et al. (2017) Fast food consumption and its associations with obesity and hypertension among children: results from the baseline data of the Childhood Obesity Study in China Mega-cities. BMC Public Health 17, 933.

16. Hare-Bruun H, Nielsen BM, Kristensen PL et al. (2011) Television viewing, food preferences, and food habits among children: a prospective epidemiological study. $B M C$ Public Health 11, 311.

17. Borghese MM, Tremblay MS, Leduc G et al. (2014) Independent and combined associations of total sedentary time and television viewing time with food intake patterns of 9to 11-year-old Canadian children. Appl Physiol Nutr Metab 39, 937-943.

18. Falbe J, Willett WC, Rosner B et al. (2014) Longitudinal relations of television, electronic games, and digital versatile discs with changes in diet in adolescents. Am J Clin Nutr 100, 1173-1181.

19. Pettigrew S, Jongenelis M, Miller C et al. (2017) A path analysis model of factors influencing children's requests for unhealthy foods. Eat Behav 24, 95-101.

20. Hong SA \& Peltzer K (2017) Dietary behaviour, psychological well-being and mental distress among adolescents in Korea. Child Adolesc Psychiatry Ment Health 11, 56.

21. Kruger AK, Reither EN, Peppard PE et al. (2014) Do sleepdeprived adolescents make less-healthy food choices? $\mathrm{Br} \mathrm{J}$ Nutr 111, 1898-1904.

22. Ferranti R, Marventano S, Castellano S et al. (2016) Sleep quality and duration is related with diet and obesity in young adolescent living in Sicily, Southern Italy. Sleep Sci $\mathbf{9}$, $117-122$

23. Weiss A, Xu F, Storfer-Isser A et al. (2010) The association of sleep duration with adolescents' fat and carbohydrate consumption. Sleep 33, 1201-1209.

24. Iaccarino Idelson P, Scalfi L \& Valerio G (2017) Adherence to the Mediterranean Diet in children and adolescents: a systematic review. Nutr Metab Cardiovasc Dis 27, 283-299. 
25. de Moraes AC, Adami F \& Falcao MC (2012) Understanding the correlates of adolescents' dietary intake patterns. A multivariate analysis. Appetite 58, 1057-1062.

26. Boone-Heinonen J, Gordon-Larsen P \& Adair LS (2008) Obesogenic clusters: multidimensional adolescent obesityrelated behaviors in the US. Ann Behav Med 36, 217-230.

27. Berkey CS, Rockett HR, Field AE et al. (2000) Activity, dietary intake, and weight changes in a longitudinal study of preadolescent and adolescent boys and girls. Pediatrics 105, E56.

28. Thivel D, Aucouturier J, Isacco L et al. (2013) Are eating habits associated with physical fitness in primary school children? Eat Behav 14, 83-86.

29. Cole T, Bellizzi M, Flegal K et al. (2000) Establishing a standard definition for child overweight and obesity worldwide: international survey. BMJ 320, 1240-1243.

30. Browning LM, Hsieh SD \& Ashwell M (2010) A systematic review of waist-to-height ratio as a screening tool for the prediction of cardiovascular disease and diabetes: 0.5 could be a suitable global boundary value. Nutr Res Rev $\mathbf{2 3}$, 247-269.

31. Council of Europe, Committee of Experts on Sports Research (1993) Eurofit: Handbook for the Eurofit Tests of Physical Fitness. Strasbourg: Council of Europe.

32. Grigorakis DA, Georgoulis M, Psarra G et al. (2016) Prevalence and lifestyle determinants of central obesity in children. Eur J Nutr 55, 1923-1931.

33. Colley RC, Wong SL, Garriguet D et al. (2012) Physical activity, sedentary behaviour and sleep in Canadian children: parent-report versus direct measures and relative associations with health risk. Health Rep 23, 45-52.

34. Paruthi S, Brooks LJ, D'Ambrosio C et al. (2016) Consensus Statement of the American Academy of Sleep Medicine on the recommended amount of sleep for healthy children: methodology and discussion. J Clin Sleep Med 12, 1549-1561.

35. Adams J, Goffe L, Brown T et al. (2015) Frequency and socio-demographic correlates of eating meals out and take- away meals at home: cross-sectional analysis of the UK national diet and nutrition survey, waves 1-4 (2008-12). Int J Behav Nutr Phys Act 12, 51.

36. Cutumisu N, Traoré I, Paquette MC et al. (2017) Association between junk food consumption and fast-food outlet access near school among Quebec secondary-school children: findings from the Quebec Health Survey of High School Students (QHSHSS) 2010-11. Public Health Nutr 20, 927-937.

37. Salvy SJ, Haye K, Bowker JC et al. (2012) Influence of peers and friends on children's and adolescents' eating and activity behaviors. Physiol Behav 106, 369-378.

38. Mikkilä V, Vepsäläinen H, Saloheimo T et al. (2015) An international comparison of dietary patterns in 9-11-yearold children. Int J Obes Suppl 5, Suppl. 2, 17-21.

39. Latorre Román PÁ, Mora López D \& García Pinillos F (2016) Feeding practices, physical activity, and fitness in Spanish preschoolers: influence of sociodemographic outcome measures. Arch Argent Pediatr 114, 441-447.

40. Bowman SA, Gortmaker SL, Ebbeling CB et al. (2004) Effects of fast-food consumption on energy intake and diet quality among children in a national household survey. Pediatrics 113, 112-118.

41. Paeratakul S, Ferdinand DP, Champagne CM et al. (2003) Fast-food consumption among US adults and children: dietary and nutrient intake profile. J Am Diet Assoc 103, $1332-1338$.

42. Sebastian RS, Wilkinson Enns C \& Goldman JD (2009) US adolescents and MyPyramid: associations between fast-food consumption and lower likelihood of meeting recommendations. J Am Diet Assoc 109, 226-235.

43. Brodney S, Mcpherson RS, Carpenter RS et al. (2001) Nutrient intake of physically fit and unfit men and women. Med Sci Sports Exerc 33, 459-467.

44. Haraldsdóttir J \& Andersen LB (1994) Dietary factors related to fitness in young men and women. Prev Med 23, 490-497. 